\title{
Male linked genomic regions determine sex in dioecious Amaranthus palmeri
}

\author{
Cátia José Neves*, Maor Matzrafi ${ }^{\dagger, * *}$, Meik Thiele*, Anne Lorant ${ }^{\dagger}$, Mohsen B. Mesgaran ${ }^{\dagger, 1}$ and Markus G. Stetter ${ }^{*, 1}$ \\ ${ }^{*}$ Institute for Plant Sciences, University of Cologne, Cologne, Germany, ${ }^{\dagger}$ Dept. of Plant Sciences, University of California, Davis, CA, USA, ${ }^{* *}$ Department of \\ Plant Pathology and Weed Research, Agricultural Research Organization, Newe Ya'ar Research Center, Israel
}

Dioecy, the separation of reproductive organs on different individuals, has evolved repeatedly in different plant families. Several evolutionary paths to dioecy have been suggested, but the mechanisms behind sex determination is not well understood. The diploid dioecious Amaranthus palmeri represents a well suited model system to study sex determination in plants. A. palmeri is one of the most troublesome weeds in the US, has successfully colonized other regions in the world and has evolved resistance to several herbicide classes. Despite the agricultural importance of the species, the genetic control and evolutionary state of dioecy in A. palmeri is currently unknown. Early cytogenetic experiments did not identify heteromorphic chromosomes. Here, we used whole genome sequencing of male and female pools from two independent populations to elucidate the genetic control of dioecy in A. palmeri. Read alignment to a close monoecious relative and allele frequency comparisons between male and female pools did not reveal significant sex linked genes. Consequently, we employed an alignment free k-mer comparison which enabled us to identify a large number of male specific k-mers. We assembled male specific contigs comprising a total of almost $2 \mathrm{Mb}$ sequence, proposing a $\mathrm{XY}$ sex determination system in the species. Based on our findings we suggest an intermediate evolutionary state of dioecy in A. palmeri. Our findings give insight into the evolution of sex chromosomes in plants and may help to develop sustainable strategies for weed management.

KEYWORDS Amaranthus, dioecy, sex chromosome, invasive weed

\section{Introduction}

$\neg$ he separation of sexes is observed in many animal and plant species, but while it is the norm in 1 animals it is very rare in plants (Bachtrog et al. 2014). In most angiosperms, individuals have both functional sex organs and are therefore either hermaphrodite, i.e. having flowers comprising the reproductive organs of both sexes, or they are monoecious, where each plant carries distinct male and female flowers. However, in a small number of plant species $(\approx 6 \%$ of known angiosperms (Renner 2014)) the female and male functions are harbored on separate individuals, i.e. they are dioecious (reviewed in Charlesworth 2016). Although rare, this breeding system has evolved independently in numerous taxonomic groups with $50 \%$ of plant families having at least one dioecious species (Renner 2014). A potential evolutionary advantage of dioecy over cosexuality is the avoidance of inbreeding and its resulting inbreeding depression. In addition, it allows the efficient allocation of resources to specialized functions of the respective sex (Charlesworth 2016).

Sex determination in dioecious plants can be environmentally or genetically controlled (Korpelainen 1998). The genetic control of sex determination in plants ranges from few individual genes (Spigler et al. 2008; Akagi et al. 2014) to non-recombining heteromorphic sex chromosomes (Sakamoto et al. 1998; Sousa et al. 2013). Most heteromorphic systems are XY male and XX female, but female specific sex chromosomes (ZW) also exist, e.g. in Silene and Salix (Slancarova et al. 2013; Pucholt et al.

${ }^{1}$ MGS: Institute for Plant Sciences, University of Cologne, Cologne, Germany - m.stetter@uni-koeln.de

MBM: Department of Plant Sciences, University of California, Davis - mbmesgaran@ucdavis.edu

Neves et al.

May 2020 
2015). Although the genetic control of dioecy has been studied in several species, the determination of sex and the evolutionary forces driving sexual dimorphism are not well understood.

Functionally, the evolution of dioecy from a cosexual ancestral state requires at least two mutations, one creating males and one creating females (Westergaard 1958; Charlesworth and Charlesworth 1978). The simultaneous emergence of both mutations is highly unlikely. Hence, full dioecy has probably evolved via intermediate states, in which some individuals are female, while the others are still cosexual (Charlesworth 2016). The second mutation, which transforms the cosexual state into males by dominantly suppressing female functions, is likely occur in close linkage to the first mutation. This reduces recombination in the genomic region, as recombinant individuals would be sterile (Charlesworth 2016). In the most common case, where males are the heterozygous sex, lack of recombination between $X$ and $Y$ chromosomes creates a male-specific region of the $Y$ chromosome, which can further result in gene degeneration (Ming et al. 2011).

As the non-recombining region spreads, further degeneration is accompanied with an accumulation of transposable elements (TEs), duplications and other mutations, leading to an increase in DNA content of the $\mathrm{Y}$ chromosome (Ming et al. 2011). In plant species with heteromorphic sex chromosomes, the Y chromosome is therefore usually bigger than their X counterpart (Charlesworth 2013; Westergaard 1958; Sakamoto et al. 1998; Sousa et al. 2013). Progressing degeneration can finally lead to massive gene loss in the $\mathrm{Y}$ chromosome and eventually a shrinkage of the sex chromosome. This last step is common in mammals (Graves 2006), and has been observed in plants (Abraham and Mathew 1962; Segawa et al. 1971), but appears to be less frequent (Bachtrog 2013). The above described signature of progressive sex chromosome evolution allows the identification of the evolutionary stage of sex determination from genomic data.

The genus Amaranthus comprises over 40 species (Sauer 1957), including crops grown for grains and vegetables (Joshi et al. 2018), as well as many invasive weeds of agricultural and natural systems (e.g. Rowland et al. 1999; Bensch et al. 2003). Most Amaranthus species are monoecious, while a few species exhibit a dioecious breeding system. In early classifications, dioecious Amaranthus species were grouped into a single subgenus (Acnida) based on their reproductive system (Mosyakin and Robertson 1996). Genome-wide phylogenetic work, however, indicated that these species cluster with monoecious species in distinct clades (Stetter and Schmid 2017). Hence, dioecy probably evolved multiple times independently within the Amaranthus genus.

Few weedy Amaranthus species are receiving increasing attention for their rapid evolution and spread of herbicide resistance (Kreiner et al. 2019; Molin et al. 2020). The dioecious A. palmeri L. is native to North America (Sauer 1957) and is one of the most devastating weeds in US agriculture (Webster and Nichols 2012; Riar et al. 2013). A. palmeri has evolved resistance to six difference classes of herbicides (reviewed in Ward et al. 2013, and www.weedscience.com) and is capable of producing copious amounts of seeds (up to 1 million per plant). Understanding the reproductive system and the sex determination of the species could help to develop agronomic strategies to decrease weed populations and mitigate herbicide resistances. Cytological analysis has shown that the chromosomes of male and female A. palmeri plants do not show heteromorphism (Grant 1959), suggesting an early stage of the evolution of dioecy in the species. Recent work using molecular markers suggested a genetic basis for sex determination in A. palmeri. However, due to low data quality and non-reproducible analyses, no inference of the evolutionary state of sex determination could be made (Montgomery et al. 2019). In addition, environmental factors can alter the expressed sex of an individual (Mesgaran et al. 2019).

In this study, we evaluated the evolutionary state of dioecy in A. palmeri to understand the genetic control and evolution of the separation of reproductive organs in plants. We show that dioecy in A. palmeri is controlled by a male specific genome region suggesting an XY system. We use high 
depth whole genome sequencing to distinguish between a sex gene system and male specific regions. Our high depth whole genome sequencing data reveals a $2 \mathrm{Mb}$ sex specific region that could not be identified through allele frequency differences when aligned to a hermaphrodite relative, suggesting the ongoing evolution of a non-recombining male specific Y chromosome in A. palmeri.

\section{Materials and Methods}

\section{Plant material}

A. palmeri seeds of two independent populations from California and Kansas were used. Seeds of A. palmeri from California (CA) and Kansas (KS) were cordially provided by Dr. Anil Shrestha (California State University, Fresno, California) and Dr. Dallas E. Peterson (Kansas State University, Manhattan, Kansas), respectively. We grew seeds from the two populations under controlled wellwatered conditions in the greenhouse in Davis, Caliofonia, USA, in the summer of 2018. About 10 seeds were planted in plastic pot $(2.37 \mathrm{~L})$, filled with a soil mix $(1: 1 \mathrm{sand} /$ peat $)$ plus a controlledrelease. Plants were grown in a greenhouse with a temperature of $32 / 22^{\circ} \mathrm{C}$ (day /night) and a day length of 16 hours provided through supplementary lighting. Seedling were thinned randomly several times to obtain one plant per pot by their four-leaf stage. Plants were irrigated through four emitters inserted into the potting medium to deliver $65 \mathrm{~mL}$ of water min-1 for two minutes and twice per day (7:00 am and 2:00 pm). We collected equal amounts of leaf tissue (3 disks of $5 \mathrm{~mm}$ diameter) of each plant once the plants were flowering and the sex could be visually determined. We pooled the leaf samples of 35 male and 32 female plants from California and 25 male and 35 female plants from Kansas for DNA extraction.

\section{Sample preparation and sequencing}

We extracted DNA from lyophilized and homogenized tissue using the DNeasy Plant Mini kit (Qiagen Sciences Inc, USA) and quantified DNA concentration on Qubit (Thermo Fisher Scientific, USA). Whole genome sequencing libraries were prepared by the UC Davis Genome Center for DNA Technologies \& Expression Analysis core facility using the TruSeq DNA library kit (Illumina, USA). After quality control, the samples were sequenced on an Illumina NovaSeq. We assessed the data quality using fastqc (https:/ / www.bioinformatics.babraham.ac.uk/projects/fastqc/) and estimated the sequence coverage based on the estimated genome size of $423 \mathrm{Mb}$ (Stetter and Schmid 2017).

\section{Allele frequency comparisons}

We aligned the raw reads to the monoecious A. hypochondiracus L. reference genome v 2.1 (Lightfoot et al. 2017) using BWA-MEM (Li 2013) and removed duplicates with picard (http:/ / broadinstitute.github.io/pi Then, we called SNP using GATK (McKenna et al. 2010) with the filter expression QD $<2.0 \quad||$ FS $>60.0$ || MQ < 40.0 || MQRankSum < -12.5 || ReadPosRankSum < -8.0 and further filtered to keep only biallelic SNPs with a maximum of 30\% missing values at a site using VCFtools (Danecek et al. 2011). We created a variant table using VariantsToTable function of GATK to test for allele frequency differences between female and male pools using the QTLseqR package (Mansfeld and Grumet 2018).

\section{Reference free $\mathbf{k}$-mer analysis}

For the reference free comparison between male and female individuals we counted unique k-mers in the male and female pool from California. We first trimmed the reads based on their quality using trimmomatic (Bolger et al. 2014) with the parameters: SLIDINGWINDOW: $4: 15$ MINLEN : 35 LEADING: 5 TRAILING: 5, before counting the occurrences 35-mers in the quality trimmed sequencing reads from 
female and male pools separately keeping only k-mers with a frequency between 15 and 2000 using Jellyfish v 2.3.0 (Marçais and Kingsford 2011). We combined the male and female counts to identify sex specific k-mers using custom scripts. We further, counted sex specific k-mers identified in the Californian population in the pools of the Kansas population using jellyfish query. To further generate the most robust set of k-mers, we filtered sex specific k-mers requiring a k-mer to have a count $\geq 50$ but $\leq 500$ within the focal Kansas (e.g., female pool for female specific) and $\leq 20$ in the Kansas opposite pool (e.g., male pool for female specific)

\section{Male specific read recovery}

We selected male specific $\mathrm{k}$-mers that had a count of minimum 50 in the male pool and 0 (represents $\leq 15$ or $\geq 2,000$, due to previous filtering) in the female pool. For all four sequenced pools, we extracted full size reads containing the k-mer sequences and their respective read pair from the trimmed read data using a custom bash script.

\section{Estimation of the size of male specific regions}

To estimate the size of the male specific region we assembled the recovered male specific reads from the male California pool with platanus-allee v 2.0.2 (Kajitani et al. 2019) using default parameters. We counted the total length of assembled base pairs as sex specific region.

\section{Extraction of male k-mer containing reads from A. tuberculatus}

We extracted the filtered set of male specific k-mers from published $A$. tuberculatus samples with known sex expression (Kreiner et al. 2019), proceeding as described above in "male specific read recovery". To normalize the numbers we devided the number of extracted reads by the total number of sequenced reads.

\section{Results}

\section{High coverage WGS of male and female pools}

We applied high depth whole genome sequencing to four pools of $A$. palmeri from two independent wild populations, separated by sex (i.e. two populations $x$ two sexes). The pools consisted of combined leaf samples of 35 male and 32 female individuals from a single population collected from California, as well as 25 male and 35 female individuals from another population collected from Kansas. The per pool sequence coverage ranged from $228 \mathrm{X}$ to $331 \mathrm{X}(322,106,487$ to $467,707,106$ reads per pool), corresponding to a mean coverage of 7.5X to 9.5X per sample (Table S1).

\section{No allele frequency differences between male and female pools}

Given the lack of cytological evidence for heteromorphic sex chromosomes in A. palmeri (Grant 1959), we tested whether sex determination in $A$. palmeri could be controlled by a specific small and still recombining region, located on an autosome. To assess this possibility, we called biallelic SNPs and compared allele frequencies between the male and female pools of the two populations and tested for frequency differences. We aligned raw reads to the reference genome of the hermaphrodite $A$. hypochondiracus (Lightfoot et al. 2017), as no reference genome of $A$. palmeri is available, yet. Overall, reads mapped to the reference genome with $90.54 \%$ to $92.55 \%$ uniquely mapped reads, indicating a high similarity between the genomes of the two species (Table S1). Yet, a large number of read pairs showed non-proper paring, indicating structural differences between the genomes.

Sex determining loci in the genome are expected to lead to significant allele frequency differences between male and female pools. We used the $G^{\prime}$ method implemented in QTLseqR to scan the first 
16 Scaffolds of the genome for significant differences in allele frequencies. Using over 12 million biallelic sites (3.2 million after filtering), distributed across the genome, we found no significant allele frequency differences between male and female pools in either of the two populations (Figs. 1 and S1). Allele frequency differences between sexes were very low across the whole aligned genome sequence.

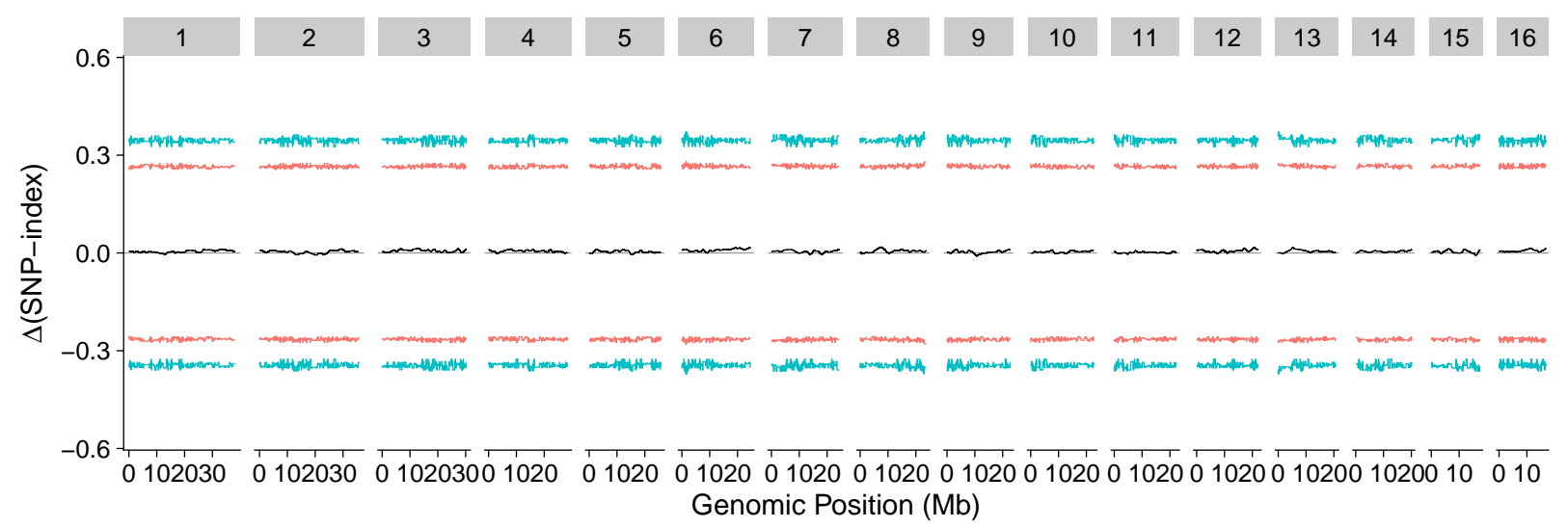

Figure 1 Differences in allele frequencies between male and female pools of the California population along the genome, relative to the A. hypochondiracus reference. Red and blue lines represent $95 \%$ and $99 \%$ confidence intervals for frequency outliers.

\section{Large number of male specific $\boldsymbol{k}$-mers}

Comparing allele frequency differences requires the successful alignment of reads to the reference genome. However, sequencing reads from diverged sex specific sequences are unlikely to align to the hermaphrodite reference genome as they accumulate large numbers of mutations (Ming et al. 2011; Charlesworth 2013). To test whether a larger, non-recombining, region could be responsible for sex determination in A. palmeri, we compared alignment free k-mer counts of male and female pools. We recovered all 35-mers (in the following referred to as k-mers) from the sequencing reads and counted the occurrences of these k-mers for each pool. Low frequency $(\leq 15)$ and high frequency $(\geq 2000)$ were discarded from the analysis as they potentially represent sequencing errors and repeat regions. We identified over 1.3 billion $(1,329,452,869)$ male and over 1.2 billion $(1,235,093,537)$ female $\mathrm{k}$-mers that passed out filters and were used for further analysis. We compared k-mer counts of the male and female pools from the California population to find k-mers that strongly deviated from the expected equal frequency in male and female pools (Fig.2). As expected, most k-mers were present in comparable numbers within males and females (Fig.2). We found 1,634,859 k-mers that were not present in females $(15 \geq n \geq 2000)$, but had a count between 50 and 500 in the male pool. We used the Kansas population to obtain the most robust set of specific k-mers. That is, we counted and identified sex specific k-mers for the California population in the Kansas population. We further filtered male (or female) specific k-mers for a minimum count of 50 and a maximum of 500 in the Kansas pool (or female). To be considered sex specific we further required a count below $20 \mathrm{k}$-mers in the female (or male) Kansas pool. After these stringent filters, we found 158,693 robust male specific k-mers, which represent $9.7 \%$ of all male specific k-mers (tab. S1). We also found a low number of female specific k-mers $(269,157)$ in the Californian population. After correction with the Kansas population, we find 2,572 female specific k-mers ( $1.0 \%$, tab S1). These include few k-mers, that are present over 2000 times in the male pools and therefore not counted in males. 


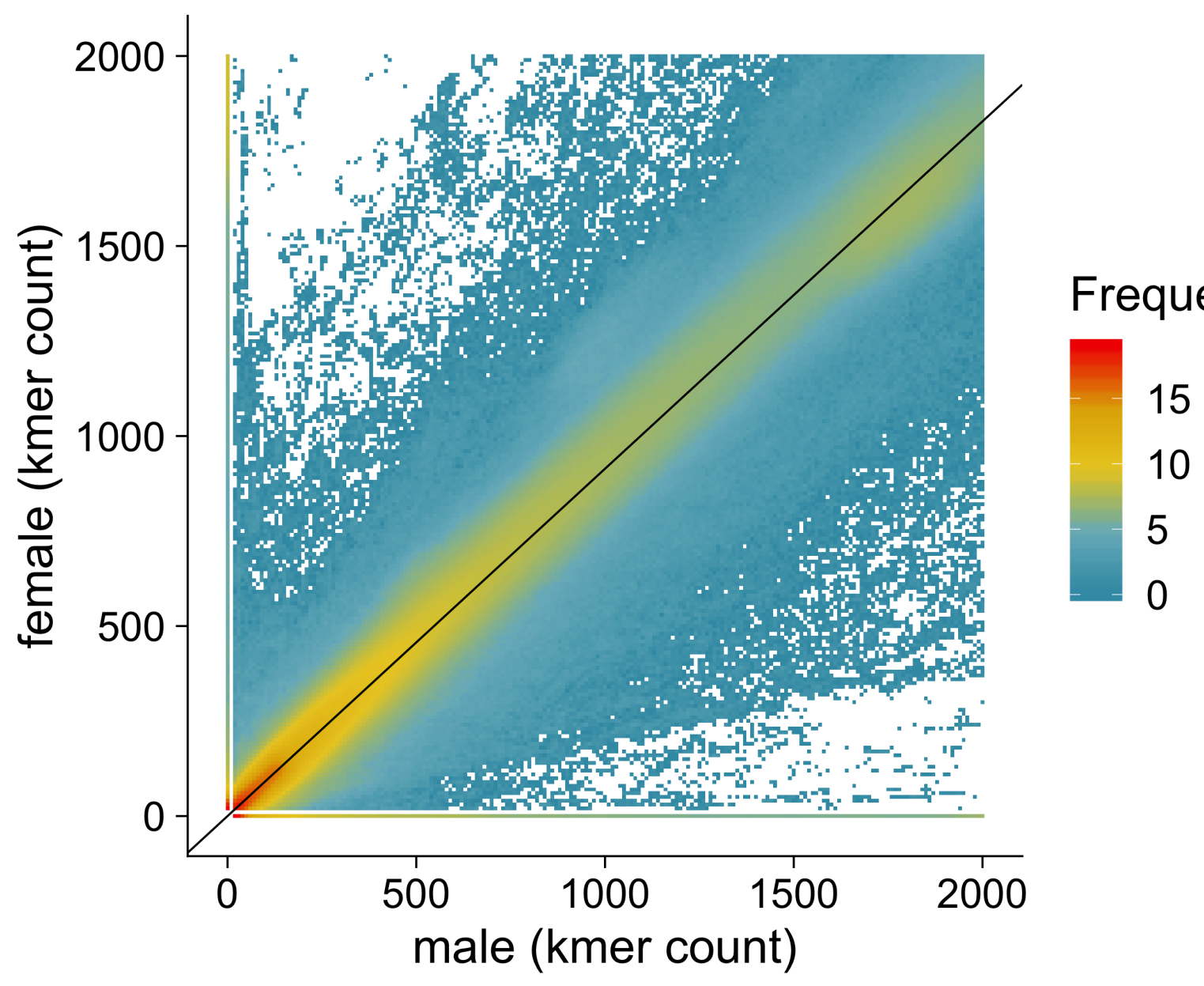

Figure 2 Comparison of the abundances of k-mers from the male and female sequence read pools of the Californian A. palmeri population. The color scale indicates the number distinct k-mers represented by one dot. The black line displays the null expectation for k-mer frequency in males and females, corrected for the number of individuals present in the male $(n=35)$ and female $(n=32)$ pool.

\section{Size estimation of the male specific region}

We extracted all reads with identified male specific $\mathrm{k}$-mers from the original reads data to assemble the male specific genome region. Only k-mers that were classified as male specific in both populations were used for the extraction of reads. The assembly process resulted in 5,774 contigs, which together consisted of 2,002,103 bp. The shortest contig was $151 \mathrm{bp}$ long and the longest scaffold was 11,654 bp long (Fig.S2). After filtering out contigs with length below 152 (maximum length of a single read), we found 3,893 contigs comprising a total length of 1,748,724 bp. Based on the estimated genome size of $423 \mathrm{Mb}$ (Stetter and Schmid 2017), the identified male specific region represents roughly $0.41 \%$ of the A. palmeri genome. 


\section{Discussion}

\section{Male specific genome region determines sex}

The emergence of sex chromosomes is an evolutionary process that starts with the occurrence of single mutations leading to unisexual individuals and ultimately giving rise to highly heteromorphic chromosomes. Our results show that the genome of $A$. palmeri harbours a male specific region, genetically controlling the sex determination. The male specific region appears to not recombine with the analogous region on the $\mathrm{X}$ chromosome and has diverged sufficiently to not align to the hermaphrodite relative. The lack of alignment to the reference sequence consequently did not allow to detect allele frequency differences between pools. Although the male sequence is strongly diverged leading to recombination suppression between male and female chromosomes in the male specific region, an accumulation of repetitive sequences seems to not have occurred, as it would have led to an increase in chromosome size that could be cytologically identified. Such a cytological difference has not been observed in A. palmeri (Grant 1959).

To further characterize the evolutionary state of sex determination in the species, we estimated the size of the male specific region. Our assembly of the region suggests approximately $2 \mathrm{Mb}$. This size likely underestimates the true size of the male specific region. Our filtering removes highly redundant k-mers, as created by transposable elements (Michael 2014). Repetitive sequence is also present in other parts of the genome and would therefore not be classified as males specific. The high number of contigs potentially results from gaps due to non-specificity of the sequence, however, the stringent filtering across two independent populations revealed high confidence sequence specific to male individuals. Fine mapping of the male specific region and identification of encoded genes will deepen our knowledge about plant sex chromosomes. In contrast to the male specific k-mers, almost all female specific k-mers found in the California sample were removed after filtering for overlaps with the Kansas population. This confirms an XY system for A. palmeri as found by male specific k-mers. However, a very low number of apparently female specific k-mers persisted after filtering. These might be involved in female reproductive functions and could be further analyzed in future molecular studies.

\section{Evolution of dioecy in A. palmeri}

The different stages of sex chromosome evolution may give us insight into the question as to when dioecy has evolved in a species. For example in the wild strawberry Fragaria virginiana male individuals coexist with females and hermaphrodites. Full dioecy is not yet evolved and the sex determining regions recombine to create unisexuals, hermaphrodites and even neuters (Spigler et al. 2008). This early stage of sex chromosome evolution indicates that the separation of sexes is rather young, whereas, the sex chromosomes in mammals and birds are very old (Cortez et al. 2014; Zhou et al. 2014). The $Y$ (mammals) and $W$ (birds) chromosomes of the heterogametic individuals of these taxa have undergone gene loss processes and finally became smaller than their $\mathrm{X}$ and $\mathrm{Z}$ chromosomes, respectively. Most heteromorphic sex chromosomes in flowering plants are increased in size as they accumulated transposable elements and other repetitive sequences. It is assumed, that this stage precedes gene loss (Ming et al. 2011). Our results show that the male specific region in A. palmeri is strongly diverged from the $X$ region whilst cytological studies have not found a dimorphism between $X$ and Y (Grant 1959). Therefore, we conclude that A. palmeri is at an early or intermediate stage of sex chromosome evolution.

The presence of cytologically homomorphic sex chromosomes that are nonetheless heteromorphic on the molecular scale has been shown in several other plant species (Liu et al. 2004; Khattak et al. 2006; Telgmann-Rauber et al. 2007), such as Spinacia oleracea, belonging to the same family as $A$. 
palmeri, i.e., Amaranthaceae.

Within the genus Amaranthus, A. palmeri and its dioecious sister species A. arenicola are placed in a monophyletic group (Stetter and Schmid 2017), in which all other species show monoecy (Wulff 1988; Costea et al. 2001; Asha et al. 2016). This suggests that dioecy has evolved independently within this group and is unlikely to be linked to the dioecious systems present in other Amaranthus species. To confirm this, we extracted reads with our set of male specific k-mers from whole genome sequencing data of A. tuberculatus individuals, another dioecious Amaranthus species, and compared the number of extracted reads (Fig. S3). We found no significant difference between male and female individuals, suggesting an independent evolution of dioecy in A. palmeri. This result agrees with the phylogentic distance between $A$. palmeri and $A$. tuberculatus which both cluster with several hermaphrodite species in genome-wide phylogenetic analyses (Stetter and Schmid 2017). Based on the phylogenetic relationship the sexual dimorphism in A. tuberculatus (and A. australis and A. floridanus) is older than dioecy in A. palmeri.

\section{Environmentally controlled sex determination}

A recent study by Mesgaran et al. (2019) showed that the sex ratio in A. palmeri plants can be changed in response to water stress. Under well-watered conditions, males and females were present in a balanced ratio, while water stress resulted in an increased female to male ratio. These findings suggest that in A. palmeri sex is not purely genetically controlled, but behaves plastic in response to environmental factors. The increase in number of females in the water stress study suggests that genetic males can change their sex expression under altered environmental conditions. Hence, sex determining loci, found in the male specific regions and their X chromosome counterpart potentially interact with autosomal genes to integrate environmental factors and alter sex determination under stress conditions.

Our work constitutes the first step in understanding the underlying processes of sex determination in A. palmeri, but the exact mechanisms and the interaction with environmental factors remain to be discovered. The male specific k-mers identified here allow to diagnose the sex of individuals in early developmental stages. This permits future studies of environmental dependent sex alteration, as it will allow to distinguish between "genetic" sex and expressed sex of an individual plant. Furthermore, the $2 \mathrm{Mb}$ male specific region can be used to fully assemble the A. palmeri $\mathrm{Y}$ chromosome. The different age and independence of dioecy evolution within the Amaranthus genus, make them a compelling system to understand the emergence of sexual dimorphism in plants. Further understanding of the sex determination in this invasive weed, can help develop novel ecological methods (e.g. shifting sex) to disrupt seed production in A. palmeri and hence reduce the impact of this invasive weed in cropping system.

\section{Acknowledgments}

We thank Julia Kreiner for sharing records of sex expression of sequenced $A$. tuberculatus samples. MBM acknowledges the support from UC Davis New Research Initiatives and Interdisciplinary Research Grant and USDA-NIFA Hatch Funding (Project No. CA-D-PLS-2511-H). We acknowledge the support of the Deutsche Forschungsgemeinschaft under Germany's Excellence Strategy - EXC2048/1 - Project ID 390686111 to MGS.

\section{Author contribution}

MBM and MGS designed and planned the study, MM and MBM provided plant material, AL performed molecular work, CJN and MGS analyzed the data. MGS supervised the study, and MT 
and MGS wrote the manuscript. All authors read and approved the manuscript.

\section{Data Availability}

Sequencing data is available from the European Nucleotide Archive (ENA) under the project numbers PRJEB38372. Scripts used for the analysis are available through figshare $10.6084 / \mathrm{m} 9$.figshare.12326306

\section{References}

Abraham, A. and P. Mathew, 1962 Cytological studies in the cycads: sex chromosomes in Cycas. Annals of Botany 26: 261-266.

Akagi, T., I. M. Henry, R. Tao, and L. Comai, 2014 A y-chromosome-encoded small RNA acts as a sex determinant in persimmons. Science 346: 646-650.

Asha, S., R. Rekha, and A. M. Sadiq, 2016 Amaranthus spinosus-a review. Bulletin of Environment, Pharmacology and Life Sciences 5: 102-107.

Bachtrog, D., 2013 Y-chromosome evolution: emerging insights into processes of y-chromosome degeneration. Nature Reviews Genetics 14: 113-124.

Bachtrog, D., J. E. Mank, C. L. Peichel, M. Kirkpatrick, S. P. Otto, et al., 2014 Sex determination: why so many ways of doing it? PLoS Biol 12: e1001899.

Bensch, C. N., M. J. Horak, and D. Peterson, 2003 Interference of redroot pigweed (Amaranthus retroflexus), palmer amaranth (A. palmeri), and common waterhemp (A. rudis) in soybean. Weed Science 51: 37-43.

Bolger, A. M., M. Lohse, and B. Usadel, 2014 Trimmomatic: a flexible trimmer for illumina sequence data. Bioinformatics 30: 2114-2120.

Charlesworth, B. and D. Charlesworth, 1978 A model for the evolution of dioecy and gynodioecy. The American Naturalist 112: 975-997.

Charlesworth, D., 2013 Plant sex chromosome evolution. Journal of experimental botany 64: 405-420.

Charlesworth, D., 2016 Plant sex chromosomes. Annual review of plant biology 67: 397-420.

Cortez, D., R. Marin, D. Toledo-Flores, L. Froidevaux, A. Liechti, et al., 2014 Origins and functional evolution of $Y$ chromosomes across mammals. Nature 508: 488-493.

Costea, M., A. Sanders, and G. Waines, 2001 Preliminary results toward a revision of the Amaranthus hybridus species complex (Amaranthaceae). Sida, Contributions to Botany pp. 931-974.

Danecek, P., A. Auton, G. Abecasis, C. A. Albers, E. Banks, et al., 2011 The variant call format and VCFtools. Bioinformatics 27: 2156-2158.

Grant, W. F., 1959 Cytogenetic studies in Amaranthus: I. cytological aspects of sex determination in dioecious species. Canadian Journal of Botany 37: 413-417.

Graves, J. A. M., 2006 Sex chromosome specialization and degeneration in mammals. Cell 124: 901-914.

Joshi, D. C., S. Sood, R. Hosahatti, L. Kant, A. Pattanayak, et al., 2018 From zero to hero: the past, present and future of grain amaranth breeding. Theoretical and Applied Genetics 131: 1807-1823.

Kajitani, R., D. Yoshimura, M. Okuno, Y. Minakuchi, H. Kagoshima, et al., 2019 Platanus-allee is a de novo haplotype assembler enabling a comprehensive access to divergent heterozygous regions. Nature communications 10: 1-15.

Khattak, J. Z., A. M. Torp, and S. B. Andersen, 2006 A genetic linkage map of Spinacia oleracea and localization of a sex determination locus. Euphytica 148: 311-318.

Korpelainen, H., 1998 Labile sex expression in plants. Biological Reviews 73: 157-180. 
Kreiner, J. M., D. A. Giacomini, F. Bemm, B. Waithaka, J. Regalado, et al., 2019 Multiple modes of convergent adaptation in the spread of glyphosate-resistant amaranthus tuberculatus. Proceedings of the National Academy of Sciences 116: 21076-21084.

Li, H., 2013 Aligning sequence reads, clone sequences and assembly contigs with BWA-MEM. arXiv preprint arXiv:1303.3997 .

Lightfoot, D., D. E. Jarvis, T. Ramaraj, R. Lee, E. Jellen, et al., 2017 Single-molecule sequencing and Hi-C-based proximity-guided assembly of amaranth (Amaranthus hypochondriacus) chromosomes provide insights into genome evolution. BMC biology 15: 74 .

Liu, Z., P. H. Moore, H. Ma, C. M. Ackerman, M. Ragiba, et al., 2004 A primitive Y chromosome in papaya marks incipient sex chromosome evolution. Nature 427: 348-352.

Mansfeld, B. N. and R. Grumet, 2018 QTLseqr: An R package for bulk segregant analysis with next-generation sequencing. The plant genome 11.

Marçais, G. and C. Kingsford, 2011 A fast, lock-free approach for efficient parallel counting of occurrences of k-mers. Bioinformatics 27: 764-770.

McKenna, A., M. Hanna, E. Banks, A. Sivachenko, K. Cibulskis, et al., 2010 The genome analysis toolkit: a mapreduce framework for analyzing next-generation DNA sequencing data. Genome research 20: 1297-1303.

Mesgaran, M. B., M. Matzrafi, and S. Ohadi, 2019 Sex lability and dimorphism in diecious palmer amaranth (Amaranthus palmeri). bioRxiv p. 769935.

Michael, T. P., 2014 Plant genome size variation: bloating and purging dna. Briefings in functional genomics 13: 308-317.

Ming, R., A. Bendahmane, and S. S. Renner, 2011 Sex chromosomes in land plants. Annual review of plant biology 62: 485-514.

Molin, W. T., A. Yaguchi, M. A. Blenner, and C. A. Saski, 2020 The eccdna replicon: A heritable, extra-nuclear vehicle that enables gene amplification and glyphosate resistance in amaranthus palmeri. The Plant Cell .

Montgomery, J. S., A. Sadeque, D. A. Giacomini, P. J. Brown, and P. J. Tranel, 2019 Sex-specific markers for waterhemp (amaranthus tuberculatus) and palmer amaranth (amaranthus palmeri). Weed Science 67: 412-418.

Mosyakin, S. L. and K. R. Robertson, 1996 New infrageneric taxa and combinations in Amaranthus (amaranthaceae). Annales Botanici Fennici 33: 275-281.

Pucholt, P., A.-C. Rönnberg-Wästljung, and S. Berlin, 2015 Single locus sex determination and female heterogamety in the basket willow (Salix viminalis L.). Heredity 114: 575-583.

Renner, S. S., 2014 The relative and absolute frequencies of angiosperm sexual systems: dioecy, monoecy, gynodioecy, and an updated online database. American Journal of botany 101: 15881596.

Riar, D. S., J. K. Norsworthy, L. E. Steckel, D. O. Stephenson, T. W. Eubank, et al., 2013 Assessment of weed management practices and problem weeds in the midsouth united states—soybean: a consultant's perspective. Weed technology 27: 612-622.

Rowland, M. W., D. S. Murray, and L. M. Verhalen, 1999 Full-season palmer amaranth (Amaranthus palmeri) interference with cotton (Gossypium hirsutum). Weed Science 47: 305-309.

Sakamoto, K., Y. Akiyama, K. Fukui, H. Kamada, and S. Satoh, 1998 Characterization; genome sizes and morphology of sex chromosomes in hemp (Cannabis sativa L.). Cytologia 63: 459-464.

Sauer, J., 1957 Recent migration and evolution of the dioecious amaranths. Evolution pp. 11-31.

Segawa, M., S. Kishi, and S. Tatuno, 1971 Sex chromosomes of Cycas revoluta. The Japanese Journal of Genetics 46: 33-39.

Slancarova, V., J. Zdanska, B. Janousek, M. Talianova, C. Zschach, et al., 2013 Evolution of sex 
determination systems with heterogametic males and females in Silene. Evolution 67: 3669-3677. Sousa, A., J. Fuchs, and S. S. Renner, 2013 Molecular cytogenetics (FISH, GISH) of Coccinia grandis: a ca. 3 myr-old species of Cucurbitaceae with the largest $\mathrm{Y} /$ autosome divergence in flowering plants. Cytogenetic and genome research 139: 107-118.

Spigler, R., K. Lewers, D. Main, and T. Ashman, 2008 Genetic mapping of sex determination in a wild strawberry, Fragaria virginiana, reveals earliest form of sex chromosome. Heredity 101: 507-517.

Stetter, M. G. and K. J. Schmid, 2017 Analysis of phylogenetic relationships and genome size evolution of the Amaranthus genus using GBS indicates the ancestors of an ancient crop. Molecular phylogenetics and evolution 109: 80-92.

Telgmann-Rauber, A., A. Jamsari, M. S. Kinney, J. C. Pires, and C. Jung, 2007 Genetic and physical maps around the sex-determining M-locus of the dioecious plant Asparagus. Molecular Genetics and Genomics 278: 221-234.

Ward, S. M., T. M. Webster, and L. E. Steckel, 2013 Palmer amaranth (Amaranthus palmeri): a review. Weed Technology 27: 12-27.

Webster, T. M. and R. L. Nichols, 2012 Changes in the prevalence of weed species in the major agronomic crops of the southern united states: 1994/1995 to 2008/2009. Weed Science 60: 145-157.

Westergaard, M., 1958 The mechanism of sex determination in dioecious flowering plants. In Advances in genetics, volume 9, pp. 217-281, Elsevier.

Wulff, R. D., 1988 Intraspecific variation in germination requirements and growth in Amaranthus dubius. American journal of botany 75: 1307-1312.

Zhou, Q., J. Zhang, D. Bachtrog, N. An, Q. Huang, et al., 2014 Complex evolutionary trajectories of sex chromosomes across bird taxa. Science 346: 1246338. 


\section{Supplement}

\begin{tabular}{lrrrr}
\hline & AMA1 & AMA2 & AMA5 & AMA6 \\
\hline \hline Population & California & California & Kansas & Kansas \\
Sex & male & female & male & female \\
Individuals & 35 & 32 & 25 & 35 \\
Raw read pairs ${ }^{1}$ & $467,707,106$ & $426,450,124$ & $322,106,487$ & $371,673,480$ \\
Per pool coverage (X) & 331.71 & 302.45 & 228.44 & 263.60 \\
Per individual coverage (mean X) & 9.48 & 9.45 & 9.14 & 7.53 \\
Mapped to A. hypochondriacus (\%) & $90.54 \%$ & $92.55 \%$ & $92.12 \%$ & $92.06 \%$ \\
Filtered paired reads ${ }^{2}$ & $460,522,536$ & $420,526,544$ & $316,496,725$ & $365,964,343$ \\
Total k-mers ${ }^{3}$ & $1,329,452,869$ & $1,235,093,537$ & $961,598,655$ & $1,066,417,174$ \\
k-mers/read pair (mean) & 2.89 & 2.94 & 3.04 & 2.91 \\
Sum male specific k-mers $^{4}$ & $17,259,830$ & 0 & $13,168,285$ & 52,698 \\
Sum female specific k-mers ${ }^{4}$ & 83,397 & 324,143 & 12,994 & 249,012 \\
\hline
\end{tabular}

Table S1 Data Summary ${ }^{1}$ Paired end $2 x 150 \mathrm{bp}$ reads. ${ }^{2}$ Trimmed and quality filtered correctly paired reads. ${ }^{3}$ 35-mers from filtered reads with $15 \leq$ count $\leq 2000 .{ }^{4}$ unique k-mers times number of each k-mer after correction with Kansas population. Values $>0$ in opposite pools result from filter steps. For instance, more than 2,000 or below 20 k-mers in opposite pool 
bioRxiv preprint doi: https://doi.org/10.1101/2020.05.25.113597; this version posted May 27, 2020. The copyright holder for this preprint (which

was not certified by peer review) is the author/funder, who has granted bioRxiv a license to display the preprint in perpetuity. It is made available under aCC-BY-NC 4.0 International license.

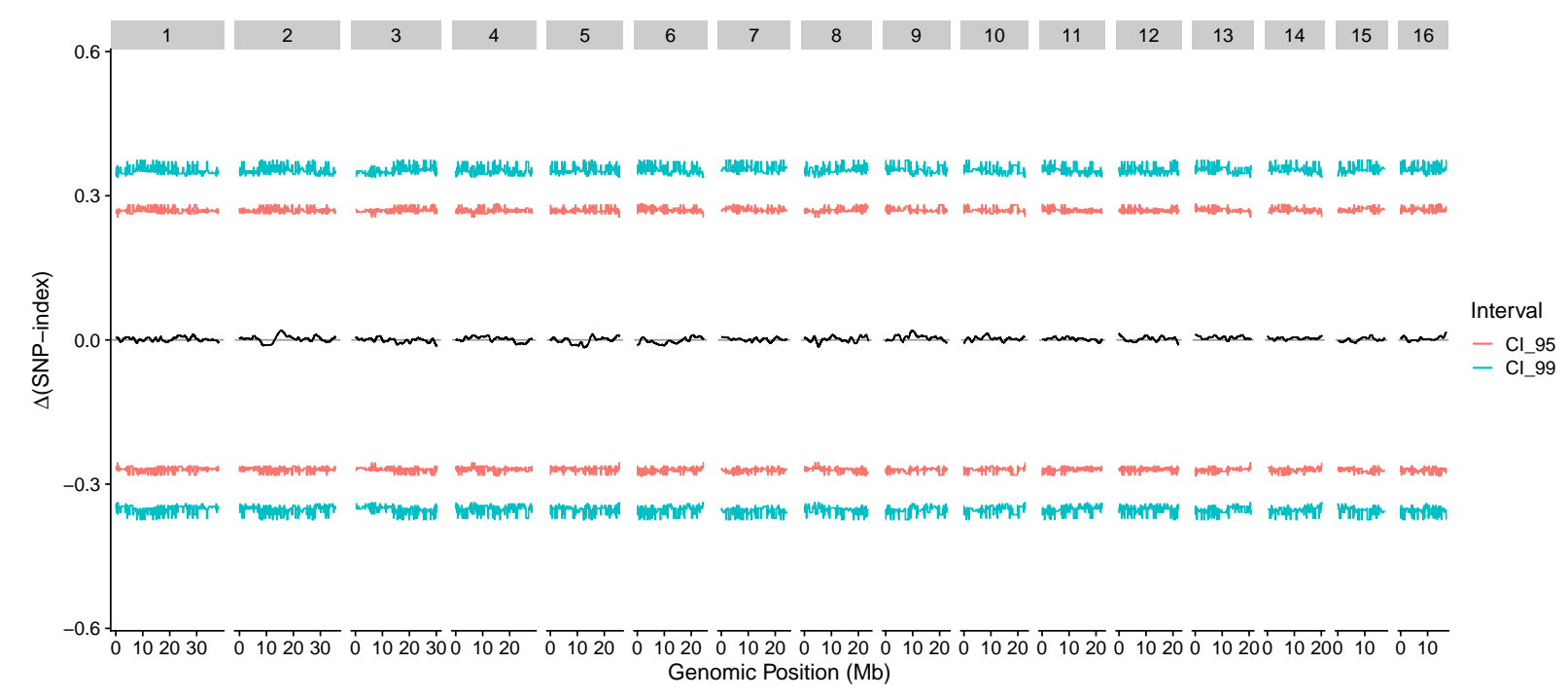

Figure S1 Differences in allele frequencies between male and female pools of the Kansas population along the genome, relative to the $A$. hypochondiracus reference. Red and blue lines represent $95 \%$ and $99 \%$ confidence intervals for frequency outliers. 
bioRxiv preprint doi: https://doi.org/10.1101/2020.05.25.113597; this version posted May 27, 2020. The copyright holder for this preprint (which

was not certified by peer review) is the author/funder, who has granted bioRxiv a license to display the preprint in perpetuity. It is made available under aCC-BY-NC 4.0 International license.

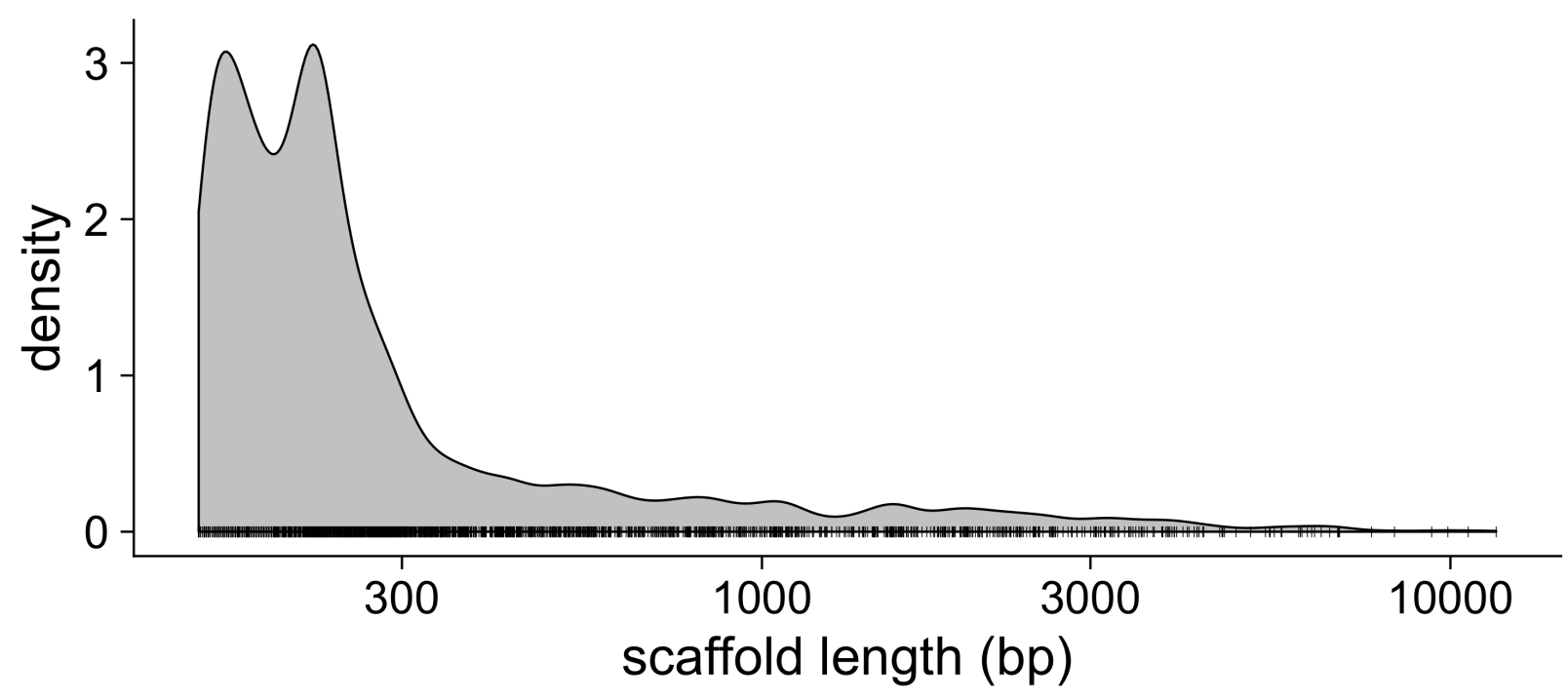

Figure S2 Scaffold size distribution Size distribution of scaffolds assembled from sex specific reads. Ticks on the $x$-axis show value distribution 


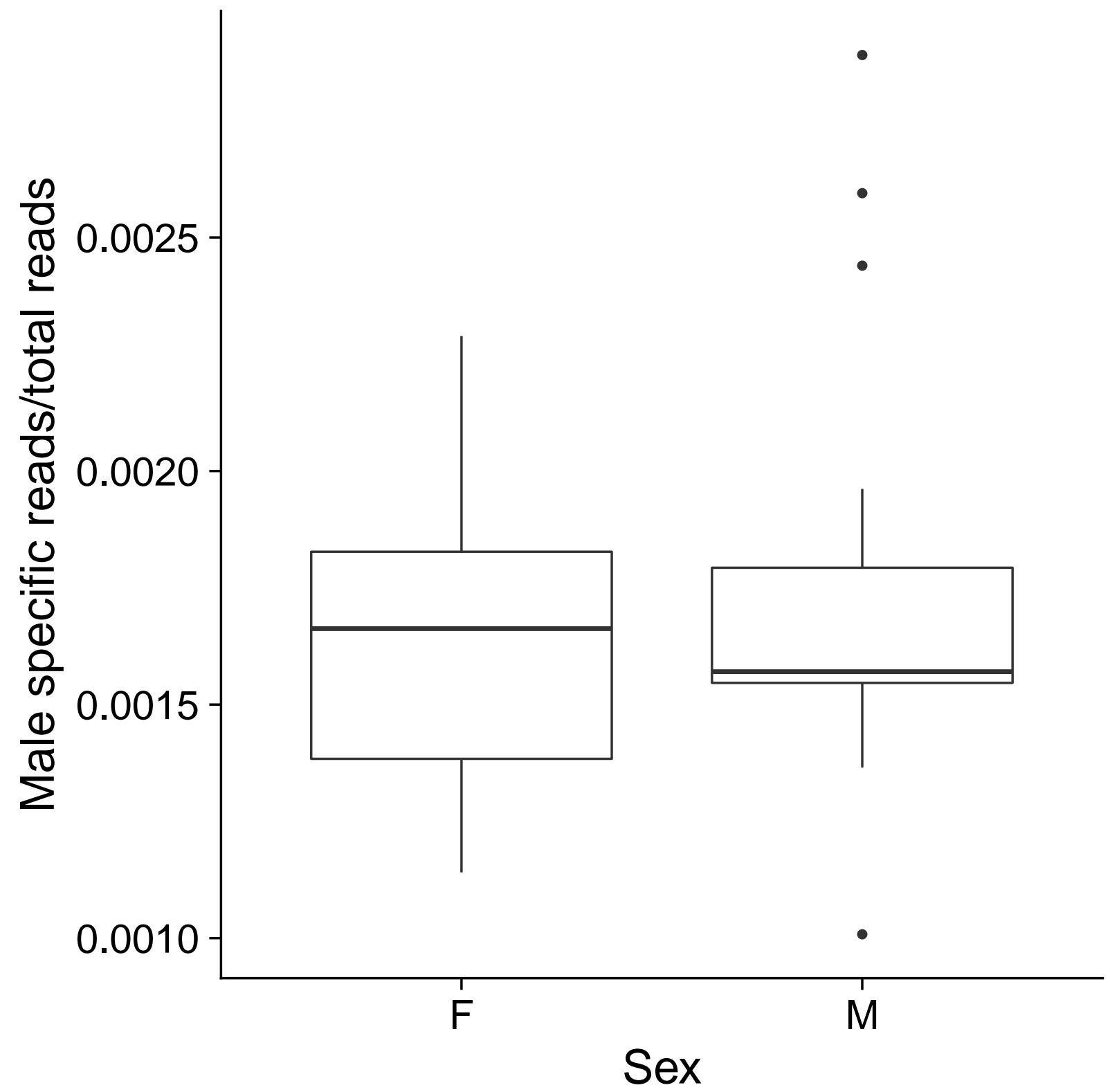

Figure S3 A. tuberculatus reads extracted Ratio of $A$. palmeri male specific k-mer containing reads in A. tuberculatus samples. No significant difference between female $(\mathrm{n}=19)$ and male $(\mathrm{n}=25)$ 